\title{
ON SOME SWITCHING INEQUALITIES OF BRENNER AND ALZER
}

\author{
C. E. M. PEARCE ${ }^{1}$ and J. PEČARIĆ ${ }^{2}$
}

(Received 17 September, 2001; revised 1 May, 2003)

\begin{abstract}
We consider some general switching inequalities of Brenner and Alzer. It is shown that Brenner's Theorem B below does not hold in general without further conditions. A simple proof is given of Alzer's Corollary D.
\end{abstract}

\section{Introduction}

It has long been known that there is an interplay between mathematical inequalities and the physical properties of networks. Thus Lehman [4] has derived a quite complicated inequality by a look-see comparison of the resistances of two electrical networks, the respective resistances representing the two sides of the relevant inequality. See also [5] for the use of Boolean functions on networks to obtain mathematical relations. Conversely we have the more common situation in which mathematical analysis may be used to establish physical properties of networks.

By considering afresh the network of [5] with the resistances replaced by switches, Turner and Conway [6] have derived the inequality

$$
\left(1-p^{n}\right)^{m}+\left(1-q^{m}\right)^{n}>1
$$

for $m, n>1$ with $p, q>0$ and $p+q \leq 1$. Here $p, q$ can be interpreted as probabilities and refer to switch reliabilities in the network. Brenner [2] has extended the domain of validity of (1.1) in the case $p+q=1$ to include $0<m, n<1$.

In a subsequent study Brenner [3] gave a refinement of (1.1) and an elaboration.

\footnotetext{
${ }^{1}$ School of Applied Mathematics, The University of Adelaide, Adelaide SA 5005, Australia; e-mail: cpearce@maths.adelaide.edu.au.

${ }^{2}$ Faculty of Textile Technology, University of Zagreb, Pierottijeva 6, 1000 Zagreb, Croatia; e-mail: pecaric@hazu.hr.
}

(C) Australian Mathematical Society 2003, Serial-fee code 1446-1811/03 
THEOREM A. Let $p$ and $q$ be positive real numbers with $p+q \leq 1$. If $m$ and $n$ are real numbers with $m, n>1$, then

$$
\left(1-p^{m}\right)^{n}+\left(1-q^{n}\right)^{m}>1+(1-p-q)^{\max (m, n)} .
$$

THEOREM B. Let $p, q, s$ and $t$ be positive real numbers, and let $k$ be a nonnegative integer. If $p+q \leq 1$ and $s \geq t>k+1$, then

$$
\sum_{i=0}^{k}\left(\begin{array}{l}
t \\
i
\end{array}\right) p^{i s}\left(1-p^{s}\right)^{t-i}+\left[1-\sum_{i=0}^{k}\left(\begin{array}{l}
t \\
i
\end{array}\right) q^{t-i}(1-q)^{i}\right]^{s}>1+(1-p-q)^{s} \text {. }
$$

It may be noted that for $k=0$ Theorem $\mathrm{B}$ reduces to Theorem A. If $p+q<1$, neither theorem holds in general for $0<m, n<1$ (see Alzer [1]).

Alzer [1] has established an elegant symmetric companion inequality to (1.3) in terms of the sum

$$
A_{k}(x, s):=\sum_{i=0}^{k}\left(\begin{array}{l}
s \\
i
\end{array}\right) x^{i}(1-x)^{s-i},
$$

where $0 \leq x \leq 1$ and $k$ is a nonnegative integer.

THEOREM C. Let $p, q, s$ and $t$ be positive real numbers, and let $k$ be a nonnegative integer. If $p+q \leq 1$ and $t, s>k+1$, then

$$
A_{k}\left(p^{s}, t\right)+A_{k}\left(q^{t}, s\right)>1+A_{k}\left((p+q)^{m}, M\right),
$$

where $m=\min (s, t)$ and $M=\max (s, t)$.

In the special case $k=0, s=m, t=n$ this provides the following improvement of (1.2).

COROllaRY D. If $m, n>1$ and $p, q>0$ with $p+q \leq 1$, then

$$
\left(1-p^{m}\right)^{n}+\left(1-q^{n}\right)^{m}>1+\left(1-(p+q)^{\min (m, n)}\right)^{\max (m, n)} \text { for } p, q>0 \text {. }
$$

In Section 2 we consider Theorem B further, while in Section 3 we address Corollary D.

\section{A counterexample to Theorem B}

Suppose we take $k=1, p=\epsilon$ (small), $q=1 / 2, s=t=5 / 2$. In the limit as $\epsilon \rightarrow 0$, the left-hand side of (1.3) tends to

$$
1+\left[1-\frac{7}{2}\left(\frac{1}{2}\right)^{5 / 2}\right]^{5 / 2}
$$


and the right-hand side to the greater value

$$
1+\left(\frac{1}{2}\right)^{5 / 2}
$$

Hence by continuity there exist positive small values of $p$ for which (1.3) fails with the values assigned to the other parameters.

Thus Theorem B is not true in general without further qualification. Hence Theorem $\mathrm{C}$ would appear to be the only established generalisation of Theorem $\mathrm{A}$.

\section{A simple proof of Corollary D}

We shall show that Brenner's proof of (1.2) can be modified to establish Alzer's refinement (1.5).

Proof OF COROLlaRY D. Define $u=1-p-q$. As in [1] set

$$
F(p):=\left(1-p^{m}\right)^{n}+\left(1-[1-p-u]^{n}\right)^{m},
$$

so that $F(p)$ is the left-hand side of (1.5). In [1], Alzer showed that $F(p)$ increases strictly to a maximum and then decreases strictly on the range $0 \leq p \leq 1-u$.

This gives for $p$ and $q$ positive that if $u>0$, then

$$
\begin{aligned}
F(p) & >\min \{F(0), F(1-u)\} \\
& =\min \left\{1+\left(1-(1-u)^{n}\right)^{m}, 1+\left(1-(1-u)^{m}\right)^{n}\right\} \\
& =1+\min \left\{\left(1-(1-u)^{n}\right)^{m},\left(1-(1-u)^{m}\right)^{n}\right\} .
\end{aligned}
$$

We now show that the last expression is equal to

$$
1+\left(1-(1-u)^{\min (m, n\}}\right)^{\max \{m, n\}} .
$$

This is immediate for $m=n$, so by symmetry we may suppose without loss of generality that $m<n$. With this assumption

$$
1-(1-u)^{n}>1-(1-u)^{m}
$$

and so a fortiori

$$
\left(1-(1-u)^{n}\right)^{m}>\left(1-(1-u)^{m}\right)^{n}
$$

from which the stated result follows.

Thus we have from (3.1) that for $p, q$ positive

$$
F(p)>1+\left(1-(p+q)^{\min \{m, n\}}\right)^{\max (m, n\}} .
$$

The desired result follows. 


\section{References}

[1] H. Alzer, "On an inequality of J. L. Brenner", J. Math. Anal. Applic. 183 (1994), 547-550.

[2] J. L. Brenner, "Some inequalities from switching theory", J. Combin. Theory 7 (1969), 197-205.

[3] J. L. Brenner, "Analytical inequalities with applications to special functions", J. Math. Anal. Appl. 106 (1985), 427-442.

[4] A. Lehman, "Problem 60-5, A resistor network inequality", SIAM Rev. 4 (1962), 150-155; 2 (1960), 152-153.

[5] A. Lehman, "A solution of the Shannon switching game", SIAM J. 12 (1964), 687-725.

[6] J. C. Turner and V. Conway, "Problem 68-I, A network inequality", SIAM Rev. 10 (1968), 107-108. 